\title{
Severe muscle cramps relieved by transcutaneous nerve stimulation: a case report
}

\author{
KR MILLS, DJ NEWHAM, RHT EDWARDS \\ From the Department of Human Metabolism, Rayne Institute, University College London School of \\ Medicine, University Street, London, UK
}

SUMMARY The case is described of a 51-year-old man with a 21 year history of severe, longlasting and widespread muscle cramps. Physical examination revealed muscle hypertrophy and fasciculation; electromyography showed spontaneously active motor units which disappeared during sleep. Cramps could be aborted by ice or mechanical vibration but the most effective management was achieved using transcutaneous nerve stimulation.

Cramp, defined as powerful, involuntary painful muscle contraction usually lasting less than one minute, is experienced by many individuals. But cramp severe enough or prolonged enough for the sufferer to seek medical advice is relatively uncommon. The symptom should be differentiated from: (a) myotonia which is usually painless, short-lasting and has characteristic electromyographic changes, (b) contracture which is painful, lasts up to several hours and is electrically silent, and (c) tetany, usually associated with hyperventilation or hypocalcaemia. ${ }^{\prime}$ Cramp is occasionally associated with dehydration, uraemia, certain drugs such as salbutamol, phenothiazines, vincristine, lithium, cimetidine and bumetanide, ${ }^{2}$ after haemodialysis and in tetanus. If the patient is truly suffering from cramp and the common associated conditions can be ruled out, then the differential diagnosis lies between "ordinary" benign cramp and the rare condition of stiff-man syndrome. A case is reported here in which severe, long-lasting, painful cramps affecting many muscle groups occurred over many years. The case appears not to conform to any of the currently described syndromes.

The mechanism of "ordinary" muscle cramp is unknown. Peripheral nerve blockade with local anaesthetic will prevent voluntary induction of a cramp, but in susceptible individuals, stimulation of the nerve distal to the block will induce a cramp. ${ }^{1}$ This suggests that the mechanism is peripheral at the

Address for reprint requests: Prof RHT Edwards, Dept of Human Metabolism, Rayne Institute, University College London School of Medicine, University St, London WC1E 6JJ.

Received 5 February 1982

Accepted 7 March 1982 muscle, but does not rule out a lesion of the peripheral nerve. The effect of neuromuscular block on "ordinary" cramp is unknown. In stiff-man syndrome, the mechanism appears to be central since peripheral nerve block arrests the painful cramp as does curarisation. Indeed, it has been suggested that the condition is due to excessive fusimotor drive to the muscle since dilute procaine (selectively blocking $\gamma$-motor axons) prevents the development of cramp.

In the case reported here, the method of treatment provided a clue to the possible mechanism. Skin stimulation at sites remote from a cramped muscle produced relief of the cramp. This is highly suggestive that a central mechanism is involved in this patient.

\section{Case report}

The patient, a 51-year-old, heavily-built, well-muscled Caucasian male began having painful cramps at the age of 30. The cramps were initially limited to the calf muscles, particularly likely to occur in the mornings on rising. Between the ages of 30 and 35 , the cramps were relatively infrequent and did not last more than 5 minutes. Gradually, the cramps became more widespread, involving the thighs, hamstrings, feet, then the shoulder girdle muscles, upper arms and small hand muscles. They also became more prolonged, lasting up to 2 hours, and would occur at any time of the day. The patient was never woken by cramp at night.

The cramps were precipitated by exercise: muscle contractions with the muscle in a shortened position such as standing on his toes would invariably produce a calf cramp; sharp, ballistic contractions such as sneezing would produce a cramp in the abdominal muscles; prolonged submaximal contractions such as in the small hand muscles in holding a telephone would also often induce a cramp as 
would passive shortening of a muscle. He was sufficiently disabled to give up his work as a builder at the age of 44 . Following a cramp, the muscle would remain sore for up to 48 hours. Myoglobinuria was never reported. No exacerbating factors were reported in relation to frequency or severity of cramps; in particular, alcohol of which he consumed moderate amounts, hot or cold weather, or dehydration did not affect the cramps. Prior to the development of cramps, he led an active life, playing water polo, boxing, weight training and at one time had been a professional footballer. His past medical history included pneumonia at the age of 10 , appendicectomy at the age of 20 and a meniscectomy at the age of 25 . No complications of the anaesthetic used in the operations were reported. About 1 year before the development of cramps he had an acute febrile illness, three days after swimming in a river, with headache, photophobia, dizziness and myalgia. He gradually recovered from this over $2-3$ weeks and a tentative diagnosis of poliomyelitis was made. There is no family history of neuromuscular disease: his father has cerebrovascular disease, but his mother is quite well. He has one brother who is of similar build with prominent calves but no symptoms. He has three children with no medical problems.

The striking abnormality on physical examination was extensive muscle hypertrophy (fig 1); this affected particularly the calves, thighs and shoulder muscles, but also the small hand muscles. Although fasciculation had been noted in his feet when he was first seen at the age of 30 , this had not been commented on in the numerous physical examinations after this. Now at the age 51, coarse fasciculation was present in both calves and thighs. The amount of fasciculation seemed to increase prior to the development of a cramp. Motor strength was striking commensurate with the increased muscle bulk. Coordination of movement and the sensory system were normal. Reflexes were symmetrical and normal, although elicitation of knee and ankle jerks would occasionally provoke a painful cramp. The plantar responses were flexor, and the cranial nerves and fundi normal. Examination of the heart, vascular system and chest revealed no abnormalities, but at various times, a liver edge up to $4 \mathrm{~cm}$ below the costal margin had been palpable.

Over the 20 year history of cramp, a variety of treatments had been tried, none with any marked success. Dantrolene sodium, diazepam, phenytoin, carbamazepine and baclofen had all been tried but were either ineffective in reducing the frequency or severity of cramp, or had to be withdrawn because of side effects. Ice packs applied to a cramped muscle were effective in reducing the duration of cramp, but could not limit the frequency. A mechanical vibrator applied to a cramped muscle would also sometimes abort a cramp. Early in the patient's investigation it had been found that electrical stimulation could abort muscle cramp and this had been used with good effect during needle muscle biopsy procedures of the quadriceps and calf muscles. Although the patient found these physical treatments useful, they were not sufficiently practical or reliable for him to use them from day to day.

Biochemical and haematological investigations were normal as were tests for thyroid and pituitary function. Serum creatine kinase examinations have occasionally

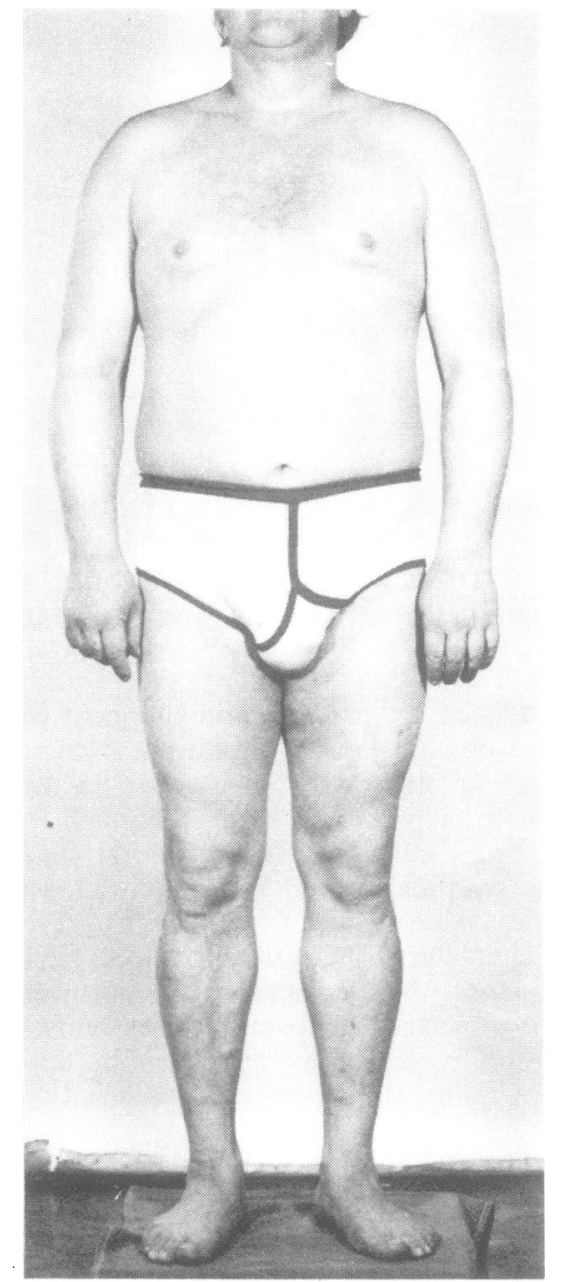

Fig 1 Muscle hypertrophy is to be seen in deltoids, wrist extensors, small hand muscles, quadriceps and calves.

been above the upper limit of normal (120 iu/l) but never above $300 \mathrm{iu} / \mathrm{l}$ and radiology of chest, skull, hands and feet were all normal as was electrocardiography.

\section{Muscle investigations}

Needle biopsy of muscle Biopsies were taken from the quadriceps and gastrocnemius muscles of the left leg using the needle biopsy technique. ${ }^{3}$ The findings were similar in both muscles; there were occasional internal nuclei and a moderate increase in perinuclear acid phosphatase. There was also a wide variation in fibre area in both fibre types.

Electromyography A concentric needle electrode (Disa type 13L49) was used to record electrical activity from the quadriceps, gastrocnemius, deltoid and abductor digiti minimi muscles. In the quadriceps, several large motor units up to $4 \mathrm{mV}$ amplitude were active at rest; this activity 
persisted over 5 minute observation and could not be abolished voluntarily by the patient even with audio feedback. The spontaneously active motor units discharged at a frequency of about $10 \mathrm{~Hz}$ and were normal in amplitude, number of phases and duration. Neither myotonia nor fibrillation was seen. On moderate volition the interference pattern was full overall amplitude up to $7 \mathrm{mV}$. In the gastrocnemius and deltoid muscles, similar activity was seen but spontaneously active motor units tended to fire at $10 \mathrm{~Hz}$ for periods of 1 minute followed by periods of silence. In the small hand muscle, no spontaneous activity was seen at rest and on moderate volition, the interference pattern was full, overall amplitude being up to $5 \mathrm{mV}$. On three occasions, overnight records of EMG activity in the left gastrocnemius muscle were made. When the patient was asleep, EMG activity which had been prominent during wakefulness disappeared. The activity reappeared very soon after waking.

Muscle strength Maximum voluntary contraction force measured by the method of Edwards $\mathrm{et} \mathrm{al}^{4}$ in the quadriceps was $890 \mathrm{~N}$ on the left and $910 \mathrm{~N}$ on the right. The predicted normal for body weight being $730 \mathrm{~N}$.

Induction of cramp in left adductor pollicis muscle After measuring the maximal voluntary contraction force of the left adductor pollicis muscle, the patient was asked to perform a $50 \%$ maximal voluntary contraction for $1 \mathrm{~s}$ every $2 \mathrm{~s}$ with the circulation occluded. After 1.75 minutes of such exercise, a cramp developed in the muscle (fig 2 ). The force developed during cramp was about $25 \%$ of the maximal force of the muscle. After about $20 \mathrm{~s}$, ulnar nerve stimulation at the wrist with $50 \mu$ s pulses at $1 \mathrm{~Hz}$ were applied with gradually increasing strength. After about $5 \mathrm{~s}$, the cramp force began to fall and had declined to zero within $20 \mathrm{~s}$. Stimuli were above the sensory threshold but there was no evidence from the force record of any motor activity.

\section{Treatment}

Because of the obvious beneficial effects of nerve stimulation on an established cramp in a small hand muscle, it was

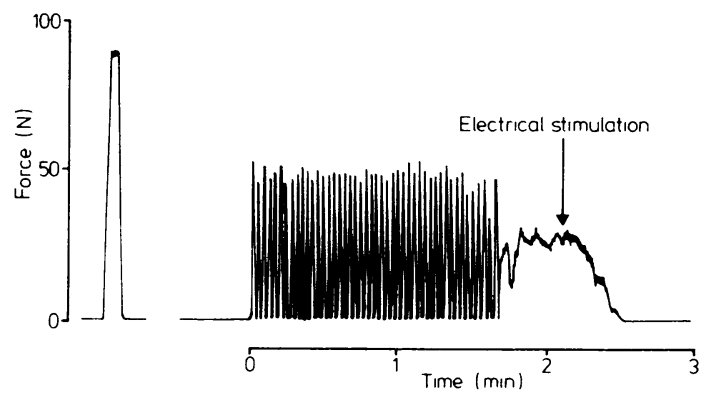

Fig 2 Force generation in the left adductor pollicis muscle during induction of cramp. 50\% maximal voluntary contractions were performed with ischaemia until cramp occurred. The cramp force is about $25 \%$ of the maximal force. Electrical stimulation of the ulnar nerve results in prompt cessation of cramp.

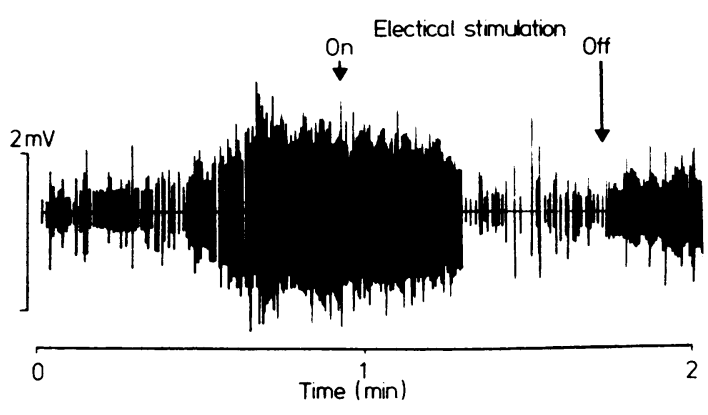

Fig 3 Surface EMG from the left gastrocnemius muscle. There is spontaneous motor unit activity at rest. This increases during the occurrence of a cramp. TNS was applied between the scapulae and electrical activity became less as the cramp resolved. Switching off TNS resulted in prompt increase in electrical activity again.

decided to try the effects of transcutaneous nerve stimulation (TNS) over the calf muscles where cramps could be induced easily by asking the patient to stand on his toes. It was found that voluntarily-induced and spontaneous cramps in the calf muscles could be aborted by skin stimulation over the muscle (fig 3 ). The stimulation was effective as long as the sensory threshold was exceeded but the patient, who controlled the voltage of stimuli, often turned the output up to above the motor threshold. The patient preferred relatively low frequency stimulation $(15-30 \mathrm{~Hz})$ as a continuous train of pulses.

To investigate whether the skin dermatome stimulated influenced the cramp, electrodes were positioned at locations successively more remote from the left calf. It was found that stimulation of the skin of the opposite calf, upper thighs of either side, and back up to a level of about the 4 th thoracic dermatome were effective in aborting cramps in the left calf. These results can only be explained by invoking a central mechanism for the cramp (see Discussion). By placing the two electrodes between the scapulae, the patient found he could abort cramps in almost any of the commonly affected muscles by turning on the skin stimulation. In addition to its effect in cramp itself, TNS also curtailed the period of muscle soreness which regularly followed a severe cramp. Before treatment, a severe calf or thigh cramp would be followed by $24-48$ hours of soreness in the muscle which would limit mobility. By applying TNS positioned between the scapulae overnight, soreness of muscles was absent the following day and mobility was normal.

\section{Discussion}

The diagnosis in this case is not clear cut. Cramps were too severe, prolonged and widespread to be classified as "ordinary", benign muscle cramps, and there was no evidence of myotonia or electolyte disturbance. The clinical criteria suggested ${ }^{5}$ for the diagnosis of "stiff-man" syndrome are: (1) continuous "board-like" stiffness of muscles, (2) paroxysms of intense muscle spasms, (3) abolition of muscle 
stiffness during sleep, (4) normal motor and sensory examination, (5) preservation of intellect. Whilst the patient discussed here certainly had paroxysms of muscle spasm, "board-like" stiffness was not found, and the physical examination revealed muscle hypertrophy. Other features reported in "stiff-man" syndrome have been constant motor unit firing at rest and abolition of this spontaneous activity during sleep both of which the patient demonstrated, but the favourable response to diazepam reported commonly in "stiff-man" syndrome was not seen. Thus while many features of this unusual syndrome are present, the cardinal symptom of continuous stiffness was absent.

Although it is impossible to conduct trials of TNS in a blind fashion and it is conceivable that cramps in this case could have a voluntary component, it is felt that the results of treatment whilst monitoring EMG are indicative of an organic cause. If this is correct then the mechanism must be in the spinal cord or cephalad to this since it is difficult to envisage a peripheral muscle mechanism in which cramps can be aborted by skin stimulation remote from the site of the cramp. The finding of continuous motor unit firing at rest is also supportive of a central state of enhanced excitability.

It is suggested that in the case discussed here, spinal motor neurons are in a state of increased excitability which sporadically manifests itself by the production of cramp. These motor neurons can be inhibited by sensory stimuli from remote areas of skin and this mechanism is responsible for the abolition of cramp by TNS. Even if the mechanism is not as outlined above, the success of treatment should be noted since other patients with severe muscle cramps of uncertain aetiology may benefit. It is salutory to note that this is the approach advocated in 1887 by Bartholow. ${ }^{6}$

We thank Dr GM Stern, Consultant Neurologist, University College Hospital, for originally referring the patient and for his helpful comments on management, and Professor PD Wall and Professor T Biscoe, University College London, for useful discussion. Financial support from the Wellcome Trust and the Muscular Dystrophy Group of Great Britain is gratefully acknowledged.

\section{References}

' Layzer RB, Rowland LP. Cramps. New Engl J Med 1971;285:31-40.

${ }^{2}$ Lane RJM, Mastaglia FL. Drug-induced myopathies in man. Lancet 1978;ii:562-6.

${ }^{3}$ Edwards RHT, Young A, Wiles CM. Needle biopsy of skeletal muscle in diagnosis of myopathy and the clinical study of muscle function and repair. New Engl $J$ Med 1980;302:261-71.

${ }^{4}$ Edwards RHT, Young A, Hosking GP, Jones DA. Human skeletal muscle function: description of tests $\frac{}{\mathbb{D}}$ on and normal values. Clin Sci Molec Med 1977;52:283-90.

${ }^{5}$ Gordon EE, Januszko DM, Kaufman L. A critical survey of stiff-man syndrome. Am J Med 1967;42:58299.

- Bartholow R. Medical Electricity; a practical treatise on the applications of electricity to Medicine and Surgery Philadelphia: Lea Bros \& Co, 1887, 3rd ed. 